\title{
Reassigned spectrum-based feature extraction for GMM-based automatic chord recognition
}

\author{
Maksim Khadkevich and Maurizio Omologo*
}

\begin{abstract}
Most existing automatic chord recognition systems use a chromagram in front-end processing and some sort of classifier (e.g., hidden Markov model, Gaussian mixture model (GMM), support vector machine, or other template matching technique). The vast majority of front-end algorithms derive acoustic features based on a standard short-time Fourier analysis and on mapping energy from the power spectrum, or from a constant-Q spectrum, to chroma bins. However, the accuracy of the resulting spectral representation is a crucial issue. In fact, conventional methods based on short-time Fourier analysis involve an intrinsic trade-off between time resolution and frequency resolution. This work investigates an alternative feature set based on time-frequency reassignment, which was applied in the past to speech processing tasks such as formant extraction. As shown in the following experiments, the reassigned spectrum provides a very accurate front-end for the GMM-based chord recognition system here investigated.
\end{abstract}

Keywords: Chord recognition; Music information retrieval; Acoustic features; Hidden Markov models; Time-frequency reassignment

\section{Introduction}

With the rapid growth of digital media and musical collections that can be accessed via the Web, many new applications are presently envisaged which require the analysis of audio contents. Automatic extraction of highlevel information such as rhythm, harmony, key, and melody therefore represents a primary goal and challenge in the music information retrieval (MIR) research community. In particular, automatic chord recognition has always been of great interest, since a chord sequence can act as a robust mid-level representation for a variety of MIR tasks such as cover song identification, music classification, and retrieval [1]. The majority of chord recognition systems proposed in the literature rely on two basic steps, namely feature extraction and classification.

Feature extraction analyzes the waveform and converts it into a set of acoustic feature vectors, which efficiently characterize the input signal in time and frequency [2]. Historically, studies on feature extraction for chord recognition started with the introduction of chroma features,

\footnotetext{
*Correspondence: omologo@fbk.eu
}

Fondazione Bruno Kessler-irst, via Sommarive 18, Povo 38050, Italy a.k.a., pitch class profiles, in 1999 by Fujishima [3]. A chroma vector represents the power spectrum distribution over the 12 semitone pitch classes for a given time interval. It is generally computed by transforming the signal from the time domain into the frequency domain, with the help of a short-time Fourier transform (STFT) $[4,5]$ or of a constant- $Q$ transform [6,7], and then mapping the energy from spectral bins to chroma bins.

Similarly to the spectrogram for speech analysis, the chromagram (i.e., a sequence of chroma vectors) is one of the most effective signal representations for music analysis and chord recognition. Different ways of computing chroma features and chromagrams are reported in the literature $[8,9]$. In particular, much attention has been devoted to the problem of higher harmonics and to their impact on chroma vectors. Several works proposed the use of some sort of harmonic analysis in order to reveal the presence of higher harmonic components [10-12]. In all these approaches, spectral analysis is performed on a frame-by-frame basis, in order to find all the pitches that occur at each time instant.

In the work of Mauch and Dixon [10], an approximate note transcription procedure was applied before calculation of a wrapped chromagram. Their technique proved to

\section{是 Springer}

(c) 2013 Khadkevich and Omologo; licensee Springer. This is an Open Access article distributed under the terms of the Creative Commons Attribution License (http://creativecommons.org/licenses/by/2.0), which permits unrestricted use, distribution, and reproduction in any medium, provided the original work is properly cited. 
be more advantageous when considering 'difficult' chords. Ueda et al. [13] showed the importance of the 'harmonic filtering' step for feature extraction. Their chord recognition system was based on harmonic/percussive sound separation, which led to the best result in the MIREX 2008 evaluation campaign (http://www.music-ir.org/mirex/ 2008/index.php/Audio_Chord_Detection_Results).

In most of the above-mentioned techniques, a power or magnitude spectrum (regardless of the related phase information) is restructured into a 12-chroma feature representation which depends on the frequency resolution of the STFT applied to the given music signal sequence. The central assumption of the STFT is that of stationarity, i.e., the spectral content of the analyzed frame is assumed to be constant. In general, small deviations from this theoretical assumption introduce tolerable artifacts. However, music is a non-stationary process, sometimes characterized by sudden changes (e.g., in the onset of a note or in attack transients of a percussive instrument) which require a high time resolution, or by quasi steadystate sequences embedding important small fluctuations (e.g., vibrato) for which a high frequency resolution would be necessary.

To increase frequency resolution enough to properly represent the harmonic contents of the signal, one should adopt a long analysis window, which introduces drawbacks due to the increased probability of having a chord change, or some note transitions, inside it. On the other hand, reducing the analysis window size does not allow for a reasonable frequency resolution. As a result, in the literature, the most widely used frame sizes to derive chroma vectors range from 180 to $740 \mathrm{~ms}$ [4,6,7,14-16]. To provide smoothed feature sequences, a high overlap ratio (i.e., from $50 \%$ to $90 \%$ ), with subsequent median filtering or averaging, is generally applied. As an alternative approach, Rocher et al. [17] proposed multi-resolution STFT analysis to estimate chords and tonality at the same time. In that work, window sizes of 800,200 , and 100 ms were jointly used to derive three versions of a chromagram.

Such techniques and related window sizes, however, are often not sufficient to avoid the above-mentioned problems. In other words, a chromagram for music signal analysis, like a spectrogram for speech analysis, is a time-frequency representation with a given uncertainty due to the adopted windowing operation, as discussed in depth in $[18,19]$. Using other time-frequency analysis techniques [20], this 'resolution trade-off' between time and frequency can be addressed to reduce uncertainty in the resulting representation.

In particular, a very accurate description of the timefrequency structure for a music signal sequence can be obtained through the reassigned spectrogram representation, which derives from the time-frequency reassignment (TFR) technique [20] initially proposed by
Kodera et al. [21]. In practice, TFR remaps the spectral energy corresponding to a spectrogram cell into another time-frequency point, which is closest to the true region of support of the analyzed signal. The resulting reassigned spectrogram has already been investigated for different tasks, such as sinusoidal synthesis [22], and cover song identification [23]. Hainsworth and Wolfe [24] showed the importance of TFR to segment a monophonic piano melody and locate partials of individual notes. As shown in this paper, TFR can also be applied effectively to enhance chroma features for chord recognition, due to its capability to highlight the energy of harmonic components.

The second main processing component of an automatic chord recognition system is classification. Possible classification approaches can here be divided in two categories: template matching $[25,26]$ and machine learning $[4,14,27]$, with hidden Markov models (HMM) being the most popular approach in, e.g., speech processing. Although the focus of this paper is on feature extraction, our work also investigates different ways of combining the HMM framework with the proposed front-end processing. In particular, in the following, we investigate the use of HMMs [28] and Gaussian mixture models (GMM). Note that the latter can be seen as a specific case of HMMs [29] and that they have already been adopted for automatic chord recognition [30].

The structure of the paper is as follows: in Section 2, the formulation of the time-frequency reassignment technique is introduced. Sections 3 and 4 describe the chord recognition system and the adopted evaluation metrics, respectively. Experimental results are then given in Section 5. Section 6 will provide a final discussion and draw some conclusions.

\section{Reassigned spectrum-based chromagram}

Let $x(n)$ be a discrete-time signal sampled at $F_{s}$ sampling rate. At a given sample $l$, the STFT is applied to $x(n)$ as follows:

$$
X(l, k)=\sum_{n=0}^{N-1} h(n) x(n+l) e^{-j 2 \pi n k / N},
$$

where $h(n)$ is the discrete version of the continuous window function $h(t)$ while $k$ and $N$ denote a bin number and the window size, respectively.

In the following, we will use the magnitude spectrum $|X(l, k)|$ deriving from Eq. (1). The majority of chromagram extraction techniques use this representation to map spectral energies to chroma bins, based on:

$$
d(k)=\bmod \left(12 \log _{2}\left(\frac{f_{k}}{f_{\text {ref }}}\right)+69,12\right)
$$

where $f_{\text {ref }}$ denotes the reference frequency of the 'A4' tone while $f_{k}=\frac{k F_{s}}{N}$ and $d(k)$ are the $k$-th frequency analyzed by the STFT and the corresponding semitone chroma bin 
index, respectively. An evident drawback of a processing based on this representation is that it neglects phase information.

On the other hand, the resulting $\operatorname{STFT} X(l, k)$ can be formulated as:

$$
X(l, k)=|X(l, k)| e^{j \phi(l, k)},
$$

where $\phi(l, k)$ is the phase spectrum.

Now, let us denote the angular frequency of $f$ as $\omega=2 \pi f$. As shown in [18] and [31], starting from a generic time-frequency point $(t, \omega)$ where STFT was computed, the reassigned time-frequency coordinates $(\hat{t}, \hat{\omega})$ can be derived in different ways.

An efficient computation of $\hat{t}(t, \omega)$ and $\hat{\omega}(t, \omega)$ in the discrete-time domain was proposed by Auger and Flandrin [32] and takes the following form:

$$
\begin{aligned}
& \hat{t}(t, \omega)=t-\Re\left\{\frac{X_{\mathcal{T} h}(t, \omega) X^{*}(t, \omega)}{|X(t, \omega)|^{2}}\right\} \\
& \hat{\omega}(t, \omega)=\omega+\Im\left\{\frac{X_{\mathcal{D} h}(t, \omega) X^{*}(t, \omega)}{|X(t, \omega)|^{2}}\right\},
\end{aligned}
$$

where $X_{\mathcal{D} h}$ is the STFT obtained using a frequencyweighted window function $h_{\mathcal{D}}(n)$ that is a discrete version of $h_{\mathcal{D}}(t)=\frac{d}{d t} h(t)$ and $X_{\mathcal{T} h}$ is the STFT obtained using a time-weighted window function $h_{\mathcal{T}}(n)$ that is a discrete version of $h_{\mathcal{T}}(t)=t h(t)$ [18]. Reallocating spectral energy from spectrogram cell $(t, \omega)$ to $(\hat{t}, \hat{\omega})$ concludes the reassignment operation. As a result, more precise estimates of spectral energy distribution are obtained.

The principle of the reassignment technique is to reallocate energy from the geometrical center of the analysis window to the 'center of gravity' of the spectral component this energy belongs to. However, the reassigned spectrogram can be noisy.

The signal has a meaningful output only in the neighborhood of an impulsive or harmonic component. In contexts for which there is no component of significant amplitude, the time-frequency reallocations can become random [33] and a large energy reassignment is generally observed. In order to obtain a better spectral representation, keeping the energy of harmonic components and deemphasizing that of noisy and impulsive components, the following condition should be met [34]:

$$
\left|\frac{\partial^{2} \phi(t, \omega)}{\partial t \partial \omega}+1\right|<A
$$

where $A$ is the tolerance factor, which defines the maximum deviation of the acceptable spectral component from a pure sinusoid.

It is worth noting that there is not an optimal value for $A$. It depends on the task and, in general, it must be determined empirically. Fulop and Fitz reported in [33] that values ranging from 0.1 to 0.2 are reasonable thresholds for speech signals. Nelson [34] reports on higher values but, in fact, the application of this theory to music signal processing requires a specific investigation, as addressed in Section 5.5. Efficient computation of $\frac{\partial \phi^{2}(t, \omega)}{\partial t \partial \omega}$ is given in [18] and can be expressed as follows:

$$
\begin{aligned}
\frac{\partial^{2} \phi(t, \omega)}{\partial t \partial \omega}= & \Re\left\{\frac{X_{\mathcal{T} \mathcal{D} h}(t, \omega) X^{*}(t, \omega)}{|X(t, \omega)|^{2}}\right\} \\
& -\Re\left\{\frac{X_{\mathcal{T} h}(t, \omega) X_{\mathcal{D} h}(t, \omega)}{X^{2}(t, \omega)}\right\},
\end{aligned}
$$

where $X_{\mathcal{T} \mathcal{D} h}(t, \omega)$ is the STFT obtained using a timefrequency-weighted window function $h_{\mathcal{T} \mathcal{D}}(n)$ that is a discrete version of $h_{\mathcal{T} \mathcal{D}}(t)=t \frac{d}{d t} h(t)[18]$.

A comparison between a spectrogram, reassigned spectrogram, and 'refined' reassigned spectrogram, for an excerpt from 'Girl' (by The Beatles) is provided in Figure 1. All spectrograms are computed using Hanning window of 192 ms with $90 \%$ overlapping. The figure shows how much 'sharper' the reassigned spectrogram representation is, when compared to the original 'blurred' spectrogram. This sharpness is due both to the benefits introduced by the application of Eqs. (6) and (7) (see Figure 1c) and to the fact that a substantial amount of energy has been reallocated to different chroma bins, as shown in Figure 1b (see also the results reported in Section 5.2).

\section{Chord recognition system}

This section introduces the basic components of the chord recognition system under study.

The first processing step aims to solve a possible problem of mis-tuning in the audio input, by estimating the reference frequency then used to extract acoustic features. This problem is here addressed by applying a method that is very similar to that discussed in our previous work [35] and in $[8,36]$.

Feature extraction is then applied to produce feature vector streams which represent the input for training or classification purposes.

Finally, the chord output sequence is produced by the decoding step, relying on an HMM framework which is addressed in the following, along with model topology and training.

\subsection{Feature vector extraction}

The estimation of the reference frequency is an essential preliminary step of feature extraction processing. In order to derive it from a musical excerpt, our system collects statistical information about peak distribution in the harmonic part of the reassigned spectrogram obtained by applying condition (6). For each peak, the distance from the nearest note frequency in the scale is derived, where the tuning system is based on $440 \mathrm{~Hz}$ for 'A4.' A peak in 


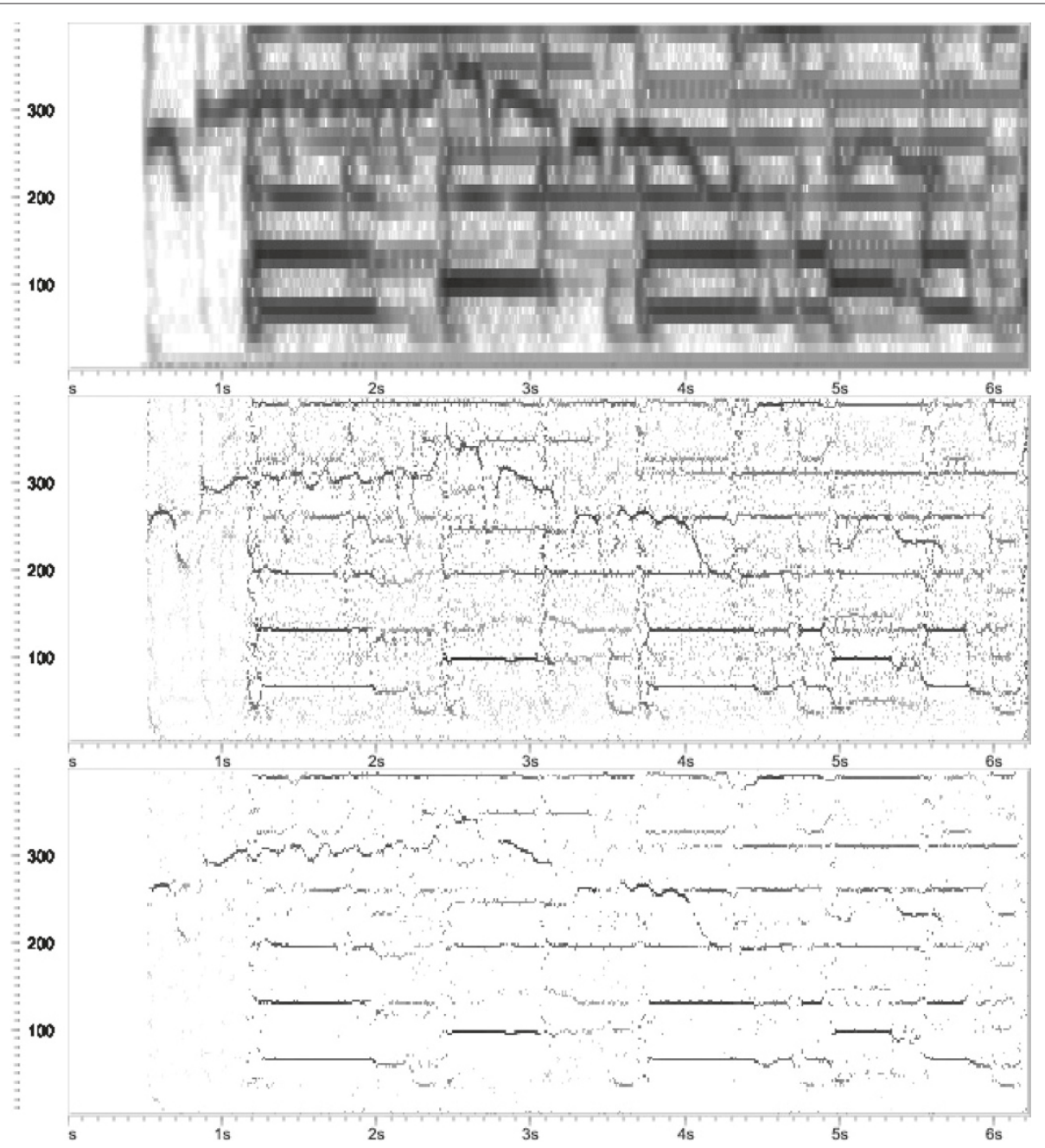

Figure 1 Time-frequency representation of an excerpt from 'Girl' (by The Beatles). From top to bottom: spectrogram, reassigned spectrogram, and harmonic reassigned spectrogram with tolerance factor set to 0.4. All spectrograms are computed using a Hanning window of 192 ms with $90 \%$ overlapping.

the distribution histogram indicates the deviation (measured in cents) of the estimated reference frequency from $440 \mathrm{~Hz}$.

Once the reference frequency is estimated, the input waveform is converted to the frequency domain by the STFT, as described in Section 2. The STFT analysis is characterized both by the size of the window applied to each analysis frame and by the overlap factor. In this work, the latter is $50 \%$, while window size ranges from 43 to 384 $\mathrm{ms}$, according to the selected feature type.

Direct folding of spectral energy to semitone pitch scale values using Eq. (2) produces standard chroma (STD) features. Applying the time-frequency reassignment technique before this procedure results in a reassigned chroma (RC) feature set. Note that only frequencies that correspond to a MIDI range between $54(185 \mathrm{~Hz})$ and 96 $(2,093 \mathrm{~Hz})$ notes are used. Harmonic reassigned chroma
(HRC) calculation is then based on the reassigned spectrum obtained when fulfilling the condition introduced in Eq. (6). The influence of the tolerance factor on system performance is investigated in Section 5.5.

\subsection{HMMs and GMMs}

HMMs have become very popular in the speech processing community since the 1980s and today, represent an established method for speech recognition applications [28] as well as for other similar contexts (e.g., speaker recognition or classification of environmental noise). Recent works have shown that HMMs also represent a very effective statistical framework for music signal analysis, in particular for chord segmentation and recognition, thanks to its ability to model sequences of acoustic events similarly to sequences of phonemes or words in speech $[27,37]$. 
In general, an HMM is characterized by a number of hidden states and a model topology. Each state is then assigned a mixture density Gaussian distribution that characterizes the statistical behavior of the feature vectors within that state of the model. In this work, feature vector components are assumed to be uncorrelated with one another, which means that the covariance matrix has a diagonal form. Finally, probabilities of self-transitions and jump-transitions between states complete the statistical characterization for a model.

As regards model topology, an HMM in which a transition is allowed between any possible pair of states is referred to as an ergodic HMM. A GMM can be viewed as a single-state HMM with a Gaussian mixture observation density, or an ergodic Gaussian observation HMM with fixed, equal transition probabilities [38]. Section 3.4 will further discuss the adoption of this model topology in automatic chord recognition.

The experimental activity reported in the following concerns the use of HMMs and GMMs, combined with TFRbased features in different ways. Figures 2 and 3 show the two main phases of the chord recognition task, i.e., training and decoding, respectively. In both cases, we assume that the input audio signal has been initially transformed into a stream of feature vectors.

\subsection{Model training}

Training of acoustic models is a fundamental step in the realization of a chord recognition system. In the literature, different approaches are proposed [14,27], where a chord is represented as a hidden state of an ergodic HMM. Similarly to the approaches of Peeters [39] and Papadopoulos et al. [5], in this work, only one model is created for each 'chord type' from the predefined dictionary. A chord type represents chords with a given set of

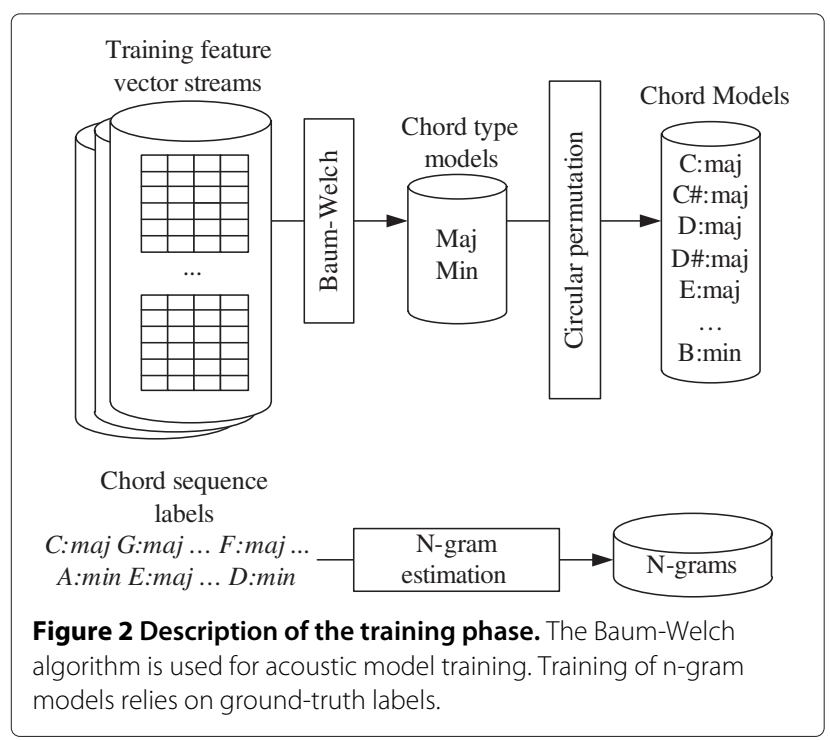

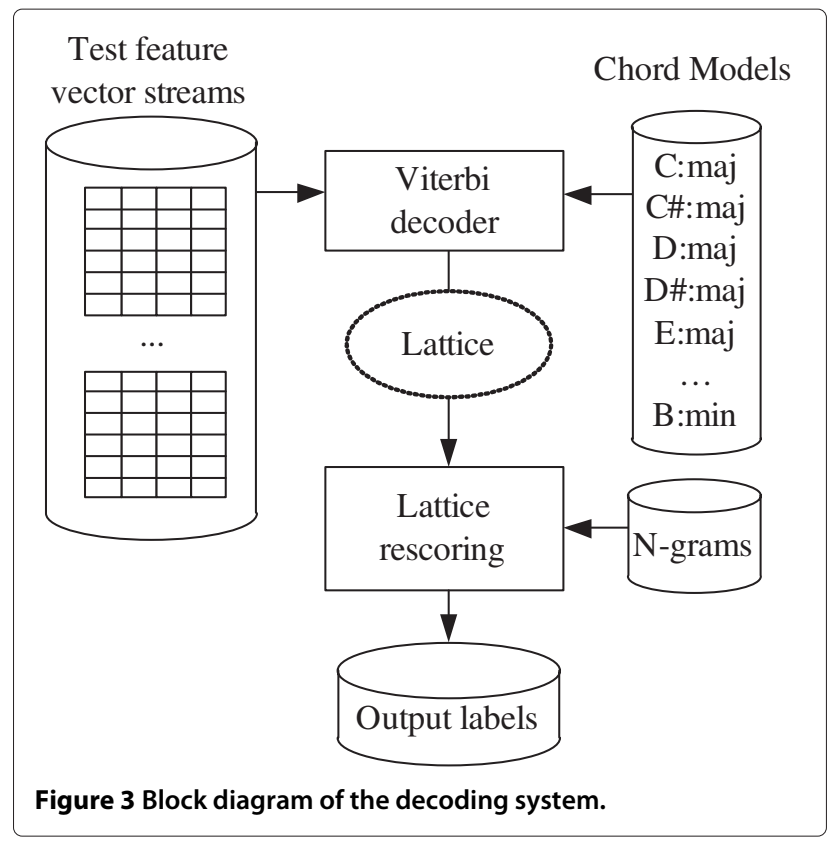

intervals between constituent notes regardless of the root note. In this work, two chord types are used, i.e., major and minor. An advantage of this approach is in terms of its flexibility when combined with a language modeling operation in a separate layer.

The final target of training is to produce 24 acoustic models (i.e., 12 for each chord type). In general, the training is based on the application of the Baum-Welch algorithm, as depicted in Figure 2.

In order to generate training material, the sequence of feature vectors is first segmented according to the groundtruth labels so that each segment contains one chord. The circular permutation [39] procedure is then applied in order to discard root information. Here, all major chords are transposed to $\mathrm{C}$ major, and all minor chords are transposed to $\mathrm{C}$ minor. For example, given chroma vectors corresponding to the A minor ground-truth chord, a circular shift by three bins is applied. A number of feature vector sequences are collected and subsequently used to train the corresponding acoustic model. Finally, in order to obtain model parameters for all possible chords related to a given chord type, another circular permutation is applied on mean vectors and the covariance matrix of multivariate Gaussians. More details on circular permutation of chroma vectors as well as on processing of means and covariance matrices are reported in [5].

Besides training of acoustic models, another important step concerns language modeling. Here, we adopt the approach described in [15], where ground-truth chord labels from the training corpus are used as an input to estimate N-gram language model parameters. Both bigram and trigram models are used here, which means that the 
probability distribution for the next chord is influenced by the one or two preceding chords.

\subsection{Model topology}

The model topology is an important choice in speech processing applications based on HMMs. The most common approach to continuous speech recognition is to select a number of context-dependent (or independent) phone models, characterized by a left-to-right topology and a limited number of states. For instance, a three-state model is often used, in which the first state refers to the beginning of the phone, the second state refers to the heart of the phone, and the third state refers to the end of the phone. The basic assumption is that the sound to model is characterized by an underlying temporal structure and that each state is assigned a Gaussian mixture observation density characterizing a specific sub-phone unit.

However, when a clear temporal structure is not evident in the speech sound to model, or when one needs to model an underlying set of hidden classes (e.g., speaker properties in the case of speaker identification), a GMM can represent a better solution. Indeed, GMMs are more commonly used than HMMs in speaker identification, as discussed in [38], and in acoustic event classification [40].

As regards chord recognition, a chord can be defined as 'a combination of notes that sound simultaneously or nearly simultaneously' [41]. However, in pop music, this concept is rather vague. Score transcription of a song is typically characterized by a compact representation consisting of a limited number of chords, each of them often corresponding to a segment in which the singer and instruments produce sequences of notes, in a nonsimultaneous way, primarily related to that chord, but with major deviations with respect to the ideal case outlined by the above-mentioned definition of chord. In this context, one cannot rely on the existence of an evident temporal structure related to the physical process characterizing all the realizations of a given chord, as is done with phone modeling for speech recognition.

As shown in Section 5.3, in the chord recognition task here addressed, no advantage is observed when using more than one state, i.e., a GMM with a given number of Gaussian mixture densities outperforms an HMM that consists of two or more states and is characterized by the same number of Gaussians.

\subsection{Decoding step}

In the decoding step, the Viterbi algorithm uses trained models that are connected in a chord loop network, as shown in Figure 4. An insertion penalty is introduced to influence the transition probability between chords. Varying the insertion penalty allows label sequences to be obtained with different degrees of fragmentation, as typically done in speech recognition tasks. As shown in

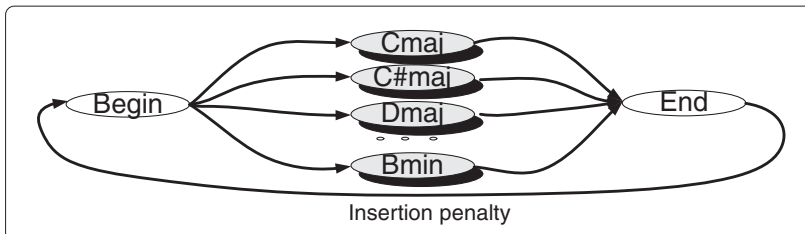

Figure 4 Chord loop grammar for the decoding step.

[15], the insertion penalty (or self-transition probability in [30]) can have a significant impact on the overall performance. For this reason, every experimental result reported in the following is based on a preliminary step with which we derived the optimal insertion penalty that maximized chord recognition performance.

As in speech recognition systems based on multiplepass decoding [42], the decoding procedure here consists of two steps. In the first step, an efficient bigram language model is applied in the Viterbi decoding, which produces a lattice. This lattice can be represented by a directed graph, where nodes denote time instants, while arcs represent different chord hypotheses. A sample lattice representation is shown in Figure 5. In the second step, the lattice obtained is rescored by applying a more sophisticated trigram language model on the reduced search space.

\section{Evaluation methodology and datasets}

\subsection{Recognition rate}

The main goal of this work is to evaluate the effectiveness of the proposed acoustic features in carrying relevant information for chord discrimination. Two chord recognition tasks are addressed in the following sections.

A simplified version of the chord recognition task is introduced in Section 5.1, where the chord loop presented in Figure 4, without transition from 'end' to 'start' states, is adopted. This means that for a given input, the recognizer outputs one of the chords included in the loop. In this case, language modeling is omitted.

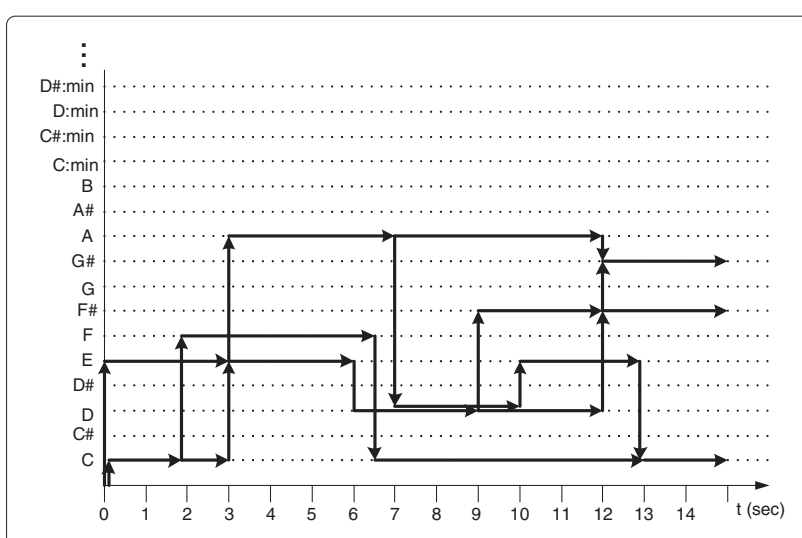

Figure 5 Example of a lattice produced in a two-step decoding process. 
For this preliminary evaluation, we used a large set of recordings of individual notes collected at the University of Iowa (http://theremin.music.uiowa.edu/MIS.html). This dataset contains high-quality note samples recorded from different instruments. Evaluation is performed based on recognition rate $(\mathrm{RR})$, in this case, computed as the total duration of correctly classified chords divided by the total duration of chords, as reported in the following:

$$
\mathrm{RR}=\frac{\mid \text { recognized_chords }|\cap| \text { ground }- \text { truth_chords } \mid}{\mid \text { ground }- \text { truth_chords } \mid} .
$$

Another recognition task is then addressed in the successive sections, which also includes the application of language modeling. In order to evaluate the chord recognition performance based on the given chroma feature sets, in this case, a threefold cross validation was accomplished on the commonly used Beatles dataset enriched with 38 songs of Queen and Zweieck. The distribution of songs into the three folds was accomplished on a random basis. The corresponding labels were kindly provided by C. Harte [43] and M. Mauch [44]. The RR measure expressed in terms of percentage is utilized as an evaluation metric. Predefined chord set consists of major and minor chord classes, resulting in 24 different chords. The 7th, min7, maj7, minmaj7, min6, maj6, 9, maj9, and min9 chords are merged to their root triads. Suspended augmented and diminished chords are discarded from this evaluation task.

\subsection{Other metrics}

Concerning the experiments on the simplified chord recognition task described in Section 5.1, in order to investigate more in-depth the properties of the proposed features, two additional criteria are used, i.e., ratio (R) and cosine measure (CM), which are computed as proposed in [45].

Let $c(n)$ be an unwrapped chroma vector extracted from a chord sample that was generated from a set of notes $e$. The $R$ estimate is the ratio of the power in the expected semitone bins, over the total power of that analysis frame. The expected semitone bins include the fundamentals and three partials for every note from set $e$.

To estimate CM, a chroma template $y(n)$ is built so that its values are set to 1 in the chroma bins that correspond to the fundamentals and to [0.66 0.330 .16 in the chroma bins that correspond to the first three overtones, respectively. The CM estimate is then computed as $\mathrm{CM}=\frac{\langle y \cdot c\rangle}{\|y\|\|c\|}$, where $\langle\cdot\rangle$ is the inner product and $\|\cdot\|$ is the $L 2$ norm.

\section{Experimental results}

In this section, we describe a series of experiments that show the convenience of using HRC features in the front-end processing. In Section 5.1, we start this study by comparing the impact of STD, RC, and HRC features on chord recognition performance in a task for which we minimize possible problems related to temporal structure, as discussed in Section 3.4. The following sections provide experimental results using the Beatles corpus, for which the latter problems generally hold. We progressively analyze different aspects that influence the behavior of the system under study, starting from the impact of TFR processing in terms of energy reallocation. Chord recognition performance is then investigated when different model topologies and window lengths are chosen, as well as when the tuning operation is performed.

Finally, Section 5.5 aims to confirm the superiority of HRC features over STD and RC ones, providing experimental results obtained both on the Beatles corpus and on the RWC data set.

\subsection{Chroma quality analysis}

In this preliminary analysis, in order to generate chord waveforms, we used the dataset of the University of Iowa that was mentioned in Section 4.1. For a given chord type, the recordings of three constituent notes are chosen from three random instruments. All the note onsets were synchronized with each other. If a note of a given instrument has a duration of more than $2 \mathrm{~s}$, everything that is after the 2nd second is discarded. Then, these samples are mixed together, producing a waveform of 2-s duration. The proposed schema for generating data results in 200 waveforms with the corresponding ground-truth information on the notes.

The obtained material is then used to evaluate the quality of different chroma features as described in the previous section. For the RR measure, half of the generated material was used as training set, and the other half was used for testing purposes. Chroma features were extracted with 192-ms window lengths, an overlapping factor of $90 \%$ and Hanning windowing.

The evaluation results for the three given chroma features are reported in Figure 6. In all the cases, $H R C$ and $R C$ significantly outperform $S T D$ feature. In particular, when comparing STD and $H R C$ features, relative improvements of $18.7 \%, 6.2 \%$, and $3.5 \%$ are observed in terms of $R, C M$, and RR, respectively. Moreover, the ratio measurements prove the ability of $H R C$ to deemphasize noise and impulsive components, which frequently occur during the note onsets. In fact, a relative improvement of $5.4 \%$ in terms of $R$ is observed comparing $R C$ and $H R C$. 


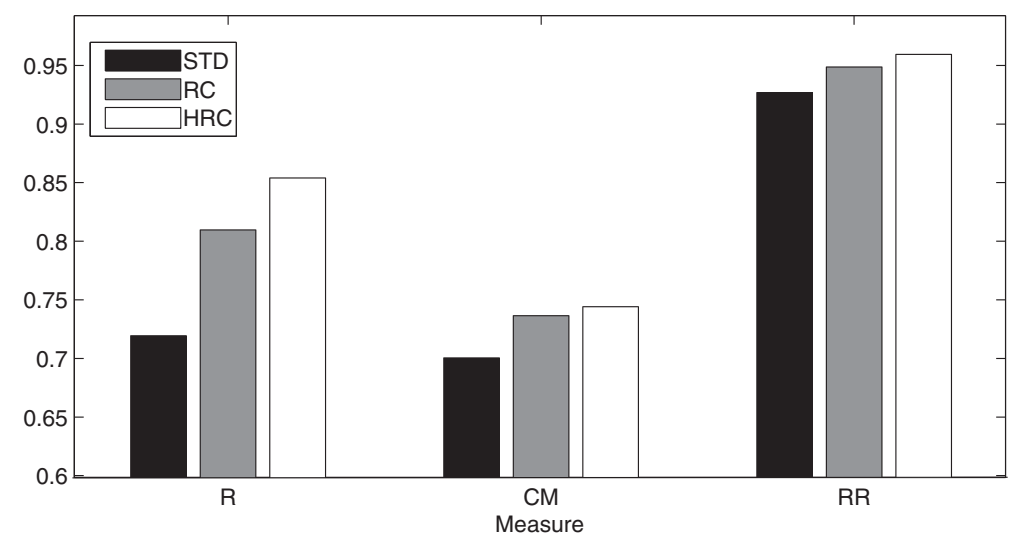

Figure 6 Chroma quality estimates. $R, C M$, and RR normalized values between 0 and 1 for STD, RC, and HRC features.

\subsection{Energy allocation in time-frequency}

In order to estimate the impact of the time-frequency reassignment operation, statistical information on the energy reallocation distance in time-frequency coordinates from the Beatles corpus was also extracted. For window length of $96 \mathrm{~ms}, \Delta f$ and $\Delta t$ distributions have zero-mean and standard deviations of $15.68 \mathrm{~Hz}$ and $14.3 \mathrm{~ms}$, respectively. The statistics of the frequency reassignments that lead to energy moving to another semitone bin are given in Table 1 . This table shows that about $9.7 \%$ of all the reassignments result in moving energy to an adjacent semitone bin, which makes an impact on the chroma energy distribution. Indeed, reallocating a substantial amount of energy between different chroma components can improve the performance of a chord recognition system.

\subsection{Number of states}

The first chord recognition experiments on the Beatles dataset regarded the analysis of performance when varying the number of states. $R R$, as a function of the total number of Gaussians for a different number of hidden states, is presented in Figure 7. The total number of

Table 1 Semitone change distribution, when using the reassigned spectrum, where frequency shift is expressed in terms of semitones

\begin{tabular}{cc}
\hline Frequency shift & Energy reassignments (\%) \\
\hline-3 & 0.14 \\
\hline-2 & 0.43 \\
\hline-1 & 4.28 \\
\hline 0 & 90.23 \\
\hline 1 & 4.33 \\
\hline 2 & 0.37 \\
\hline 3 & 0.19 \\
\hline
\end{tabular}

Gaussians, which can be calculated as number of Gaussians per state multiplied by the number of states, represents the model complexity. Experimental results showed that using a one-state model leads to the highest RR for a given model complexity. Given this, all the next experiments will be conducted using a GMM, i.e., a one-state HMM topology. For a detailed analysis on the application of more complex HMM and GMM models, including full covariance matrices, one can refer to the work of Cho et al. [30], which also shows a limited advantage in using statistical models with higher complexity.

\subsection{Influence on performance of tuning and window length}

As mentioned in the previous sections, an important step in the feature extraction process is the estimation of the deviation of the A4 note from $440 \mathrm{~Hz}$ and subsequent compensation for mis-tuning [35]. Results with STD, RC,

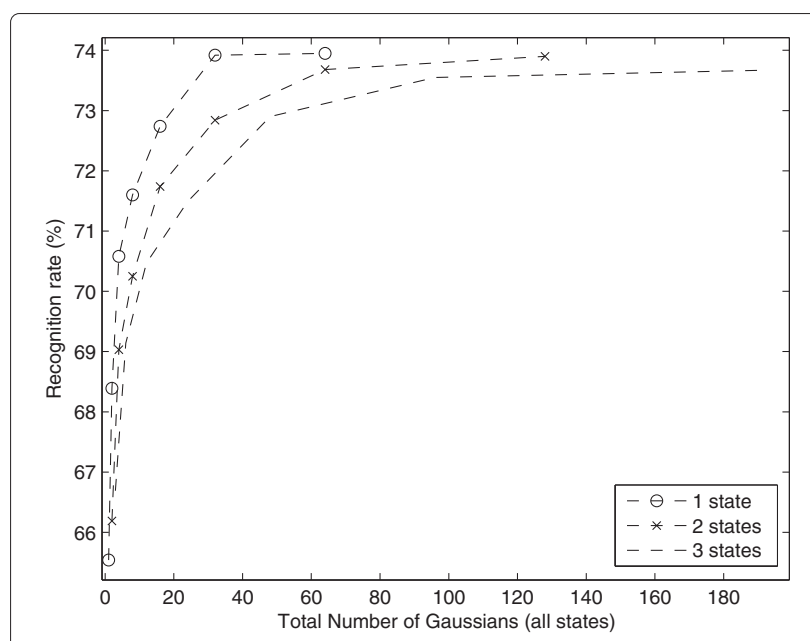

Figure 7 Recognition rate for HRC feature as a function of the total number of Gaussians. Performance is shown for a different number of hidden states in HMM. 
and HRC features, with and without tuning, are provided in Table 2.

These experimental results show that the tuning operation plays an important role and leads to an increase in performance for all the features. Moreover, the results show the advantage of adopting HRC features, which will be investigated in more detail in the following section.

Since the time-frequency reassignment technique used here includes the application of windowing, the impact of different window types on the performance was also investigated. Here, we will only report on the influence of window length on chord recognition rate, since preliminary experiments using different window types (i.e., Hanning, Hamming, Kaiser, and Blackman) had showed that no significant difference in performance is observed for any of the investigated features, i.e., STD, RC, and HRC. Similar results were obtained in [35]. Given this experimental evidence, a Hanning window is adopted in all the following experiments.

Figures 8 and 9 report on recognition rates using $R C$ and STD features with different window lengths and numbers of Gaussians. For each configuration, the performance was derived using the best insertion penalty, as outlined in Section 3.5. These results show that an optimal window length for the RC feature set is $96 \mathrm{~ms}$, as opposed to the STD feature, for which such a short window length would provide a much lower performance. This fact is coherent with a more accurate energy localization in time for the TFR-based features. For the sake of conciseness, a similar figure regarding the use of HRC is not reported, since the recognition rate is always higher than the corresponding one reported in Figure 8 for the $\mathrm{RC}$ feature. In other words, the trend of performance is similar for any of the selected window lengths, suggesting that it should be set to $96 \mathrm{~ms}$ also when $\mathrm{RC}$ features are used.

\subsection{Harmonic reassigned chroma}

As shown in Table 2, adopting HRC features improves the chord recognition rate. The purpose of this section is to provide more details on this experimental evidence.

First of all, the impact of the tolerance factor $A$ introduced in Eq. (6) was investigated, as shown in Figure 10. The optimal value of $A$ for this chord recognition task turns out to be 0.4 , with a recognition rate of $78.28 \%$, although small deviations from this setting have a minor impact in terms of loss of performance.

Table 2 Influence of tuning on chord recognition performance, using STD and RC features

\begin{tabular}{cccc}
\hline & STD & RC & HRC \\
\hline No tuning & 70.33 & 76.70 & 77.59 \\
\hline Tuning & 71.29 & 77.29 & 78.28 \\
\hline
\end{tabular}

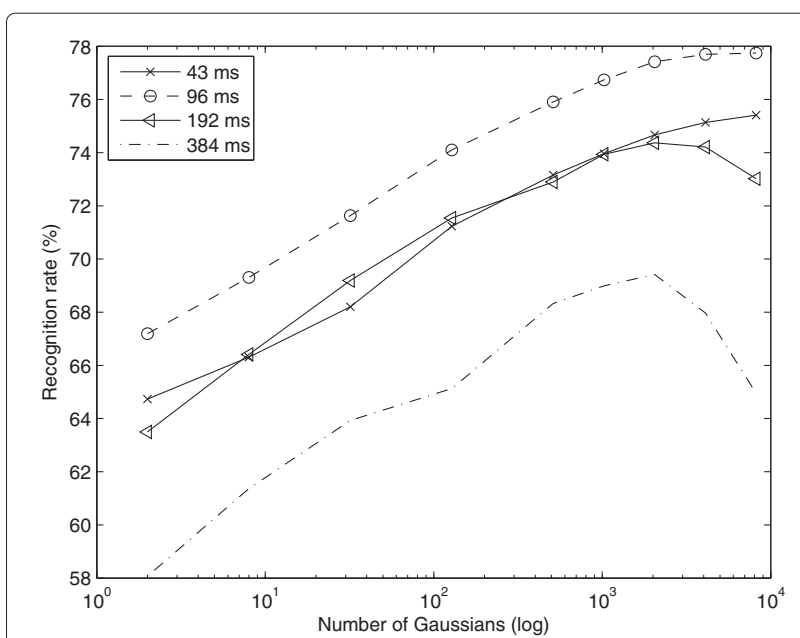

Figure 8 Recognition rates using RC features for different window lengths and Gaussian numbers.

The next set of experiments aimed to compare HRC, $\mathrm{RC}$, and STD features when varying the complexity and accuracy of the models. Figure 11 depicts recognition rates for different numbers of Gaussians. In all three cases, the results indicate that 2,048 is a good choice in terms of number of Gaussians. Higher values would not bring significant improvements and would increase the computational load. This experimental evidence, however, may depend on the task and, in particular, on the size of the training material.

In order to understand the impact of the training material on the result, another set of experiments was carried out using the RWC dataset [46], which consists of 100 pop songs. The RWC dataset was used for training, while the same Beatles dataset was used for testing. Table 3

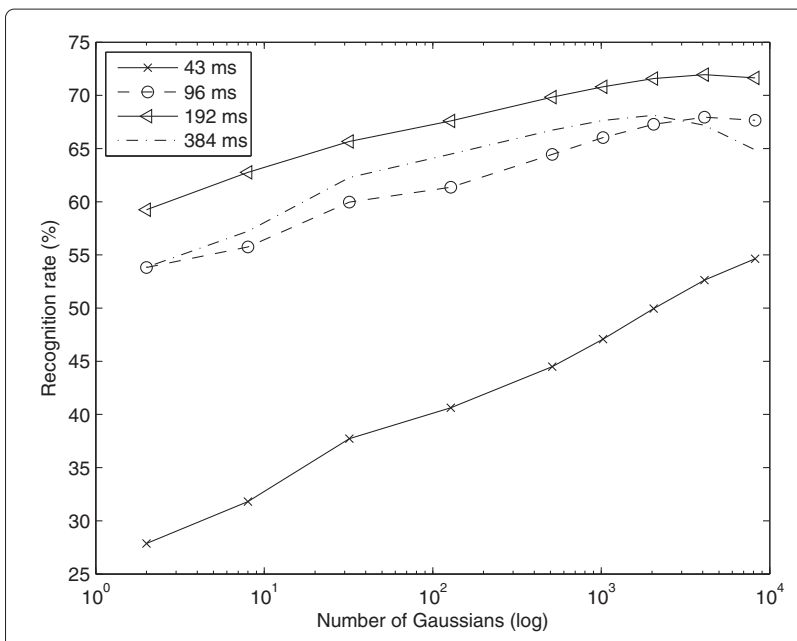

Figure 9 Recognition rates using STD features for different window lengths and Gaussian numbers. 


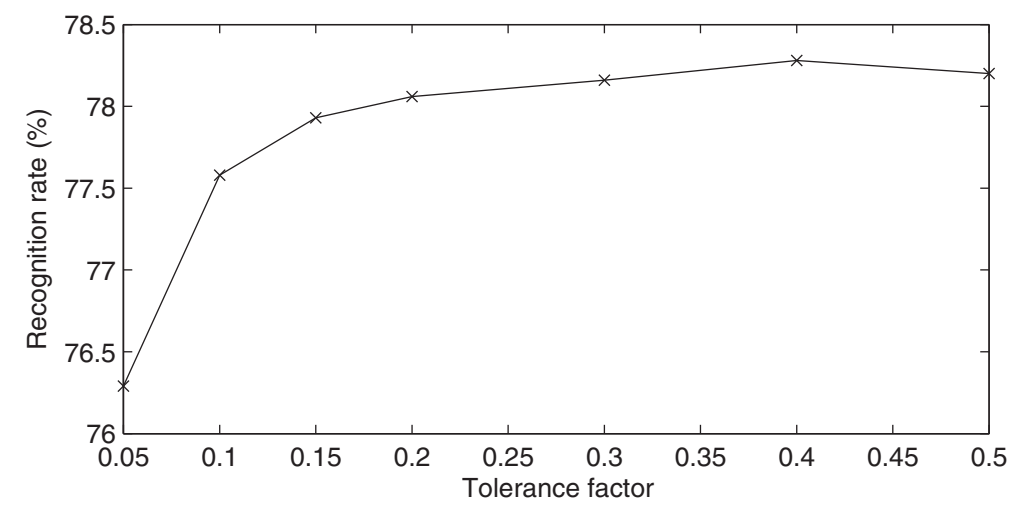

Figure 10 Recognition rate for $\mathrm{HRC}$ as a function of the tolerance factor.

reports on recognition rates for 32, 512, and 2,048 Gaussians taken from results presented in Figure 11, as well as results obtained by training models on the RWC dataset.

Note that in speech recognition, a mismatch between training and testing conditions often leads to a significant loss of performance. Our experiments show that performance is obviously lower when chord models are trained on the RWC dataset. However, the loss of performance due to this mismatch is not substantial (i.e., a reduction of about $2 \%$ to $3 \%$ ), which confirms that the proposed system seems to be quite robust to different possible choices of training material.

Moreover, it is worth noting that the advantage of using TFR over STD features is confirmed when models are trained on the RWC dataset. Also in this case, HRC features always outperform RC ones.

\section{Conclusions}

This paper investigates the important role that acoustic feature extraction plays in automatic chord recognition.

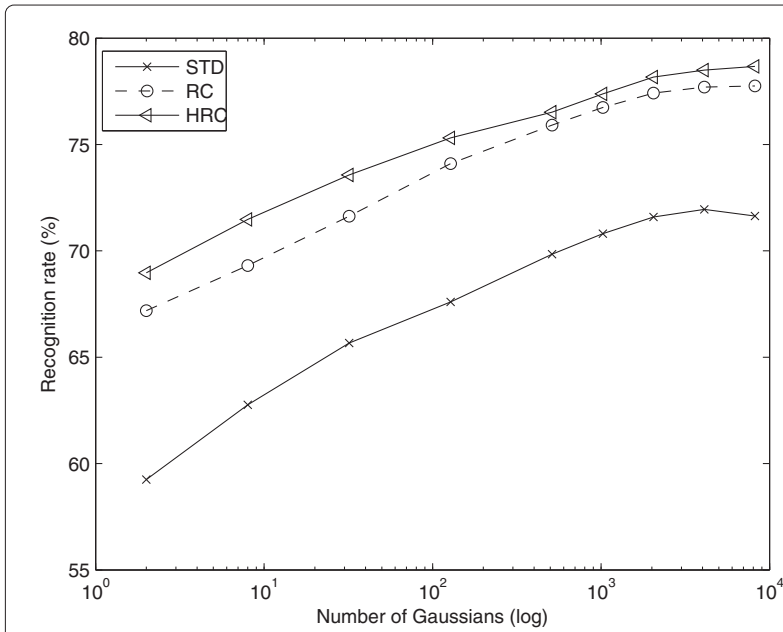

Figure 11 Recognition rate as a function of the number of Gaussians. Performance is shown for the three different feature sets, STD, RC, and HRC.
The chord recognition system under study is based on a probabilistic approach to statistically model sequences of chroma features.

The main focus of the work is on the reassigned spectrogram. This time-frequency representation is here proposed as alternative to the standard STFT to derive a more accurate set of chroma features. Experimental results showed that better chord recognition rates can be obtained with TFR-based features, if compared to performance provided by traditional STFT-based chroma features. Applying harmonic component separation in the spectral domain further increases this performance. The experimental section comprises a quantitative analysis which shows the effectiveness of time-frequency reassignment from different perspectives and with a different assignment to some key parameters.

As final result, a substantial improvement over the baseline system was obtained using the HRC feature, with a $78.28 \%$ recognition rate on a commonly used dataset. Note that an extended version of the same chord recognition system, but with additional bass chroma feature and multi-stream HMMs, was then adopted in the MIREX 2011 evaluation, in which it provided one of the best performances in terms of overlap ratio, which was equal to $82.85 \%$ (http://nema.lis.illinois.edu/nema_ out $/$ mirex2011/results/ace/).

Table 3 Recognition rates using the RWC dataset for training and the Beatles dataset for test (RWC), compared to performance obtained training on a threefold cross validation of the Beatles dataset (Beatles)

\begin{tabular}{lrrr}
\hline Number of Gaussians & $\mathbf{3 2}$ & $\mathbf{5 1 2}$ & $\mathbf{2 , 0 4 8}$ \\
\hline STD (Beatles) & 65.67 & 69.84 & 71.29 \\
\hline RC (Beatles) & 71.63 & 75.91 & 77.29 \\
\hline HRC (Beatles) & 73.57 & 76.52 & 78.28 \\
\hline STD (RWC) & 63.67 & 66.42 & 68.2 \\
\hline RC (RWC) & 69.85 & 72.5 & 74.51 \\
HRC (RWC) & 71.89 & 74.95 & 75.89 \\
\hline
\end{tabular}


Overall, this study also represents a starting point to explore different research topics.

Our experimental activity demonstrated that using a one-state HMM, i.e., GMM, leads to better performance than using two or more states. This fact probably depends on the characteristics of the training and testing material, in particular on its labeling. While the purpose of this work was to use an HMM as a framework to analyze the convenience of using TFR features, the abovementioned issue would definitely deserve a specific study based on a different definition of the chord recognition problem as well as on the adoption of other labeled music material.

As shown by the results of the MIREX 2011 chord detection evaluation campaign, multi-stream HMMs represent another possible area to further investigate, in order to better characterize the frequency band splitting and the related optimal weighting of each stream.

Another direction regards the introduction of rhythm analysis in the given framework. The most straightforward possible improvement can be obtained by including a probabilistic modeling of the temporal structure. This can be done by embedding an additional hidden layer in HMMs, as proposed in [47], where hidden states correspond to different beat phases.

Finally, an interesting research issue to investigate, in order to improve the quality of feature vectors when using TFR, is the application of a more accurate analysis of higher harmonics. For instance, one could adopt the higher harmonic subtraction technique outlined in $[10,11]$.

For most of the outlined directions, more complex chord recognition tasks will be addressed by both increasing the size of the chord set and processing different music styles (e.g., jazz).

\section{Abbreviations}

CM: Cosine measure; GMM: Gaussian mixture model; HMM: Hidden Markov model; HRC: Harmonic reassigned chroma; MIR: Music information retrieval; $R$ : Ratio; RC: Reassigned chromal; RR: Recognition rate; STD: Standard chroma; STFT: Short-time Fourier transform; SVM: Support vector machine; TFR: Time-frequency reassignment.

\section{Competing interests}

Both authors declare that they have no competing interests.

Received: 20 July 2012 Accepted: 10 June 2013

Published: 27 June 2013

\section{References}

1. JP Bello, in Proceedings of the International Conference on Music Information Retrieval (ISMIR). Audio-based cover song retrieval using approximate chord sequences: testing shifts, gaps, swaps and beats (Philadelphia, (2007), pp. 239-244

2. LR Rabiner, RW Schafer, in Introduction to Digital Speech Processing. Now Publishers Inc. (Hanover, 2007)

3. T Fujishima, in Proceedings of the International Computer Music Conference (ICMC). Realtime chord recognition of musical sound: a system using common lisp music (Beijing, 1999), pp. 464-467
4. A Sheh, DP Ellis, in Proceedings of the International Symposium on Music Information Retrieval (ISMIR). Chord segmentation and recognition using EM-trained hidden Markov models (Baltimore, 2003)

5. H Papadopoulos, G Peeters, in Proceedings of the International Workshop on Content-Based Multimedia Indexing (CBMI). Large-scale study of chord estimation algorithms based on chroma representation and HMM (Bordeaux, 2007), pp. 53-60

6. M Mauch, S Dixon, in Proceedings of the International Conference on Music Information Retrieval (ISMIR). A discrete mixture model for chord labelling (Philadelphia, 2008), pp. 45-50

7. H Papadopoulos, G Peeters, Joint estimation of chords and downbeats from an audio signal. IEEE Trans. Audio, Speech, Lang, Process. 19, 138-152 (2011)

8. M Müller, S Ewert, in Proceedings of the International Conference on Music Information Retrieval (ISMIR). Chroma toolbox: MATLAB implementations for extracting variants of chroma-based audio features (Miami, 2011)

9. M Stein, M Schubert, BM Gruhne, G Gatzsche, M Mehnert, in Proceedings of the 126th Convention of the Audio Engineering Society (AES). Evaluation and comparison of audio chroma feature extraction methods (Munich, 2009)

10. M Mauch, S Dixon, in Proceedings of the International Conference on Music Information Retrieval (ISMIR). Approximate note transcription for the improved identification of difficult chords (Utrecht, 2010), pp. 135-140

11. M Varewyck, J Pauwels, JP Martens, in Proceedings of the 16th ACM international conference on Multimedia. A novel chroma representation of polyphonic music based on multiple pitch tracking techniques (New York, 2008), pp. 667-670

12. E Gómez, P Herrera, in Proceedings of the 25th International AES Conference. Automatic extraction of tonal metadata from polyphonic audio recordings (London, 2004), pp. 74-81

13. Y Ueda, Y Uchiyama, T Nishimoto, N Ono, S Sagayama, in Proceedings of the IEEE International Conference on Acoustics, Speech and Signal Processing (ICASSP). HMM-based approach for automatic chord detection using refined acoustic features (Dallas, 2010), pp. 5518-5521

14. K Lee, M Slaney, Acoustic chord transcription and key extraction from audio using key-dependent HMMs trained on synthesized audio. IEEE Trans. Audio, Speech, Lang. Process. 16(2), 291-301 (2008)

15. M Khadkevich, M Omologo, in Proceedings of the International Conference on Music Information Retrieval (ISMIR). Use of hidden Markov models and factored language models for automatic chord recognition (Kobe, 2009), pp. 561-566

16. M Khadkevich, M Omologo, in Proceedings of the IEEE International Conference on Acoustics, Speech and Signal Processing (ICASSP). Time-frequency reassigned features for automatic chord recognition (Prague, 2011), pp. 181-184

17. T Rocher, M Robine, P Hanna, L Oudre, in Proceedings of the International Conference on Music Information Retrieval (ISMIR). Concurrent estimation of chords and keys from audio (Utrecht, 2010), pp. 141-146

18. KR Fitz, SA Fulop, A unified theory of time-frequency reassignment. Comput. Res. Repository (CoRR). abs/0903.3080 (2009)

19. PJ Loughlin, KL Davidson, Modified Cohen-Lee time-frequency distributions and instantaneous bandwidth of multicomponent signals. IEEE Trans. Signal Process. 49(6), 1153-1165 (2001)

20. SA Fulop (Ed), Speech Spectrum Analysis. (Springer, Heidelberg, 2011)

21. K Kodera, R Gendrin, C Villedary, Analysis of time-varying signals with small BT values. IEEE Trans Acoustics, Speech Signal Process. 26, 64-76 (1978)

22. T Abe, M Honda, Sinusoidal model based on instantaneous frequency attractors. IEEE Trans. Audio, Speech Lang, Process. 14(4), 1292-1300 (2006)

23. DPW Ellis, GE Poliner, in Proceedings of the IEEE International Conference on Acoustics, Speech and Signal Processing (ICASSP). Identifying 'Cover Songs' with chroma features and dynamic programming beat tracking (Honolulu, 2007), pp. 1429-1432

24. SW Hainsworth, PJ Wolfe, in Proceedings of the International Computer Music Conference (ICMC). Time-frequency reassignment for music analysis (Havana, 2001), pp. 14-17

25. L Oudre, Y Grenier, C Févotte, in Proceedings of the International Conference on Music Information Retrieval (ISMIR). Template-based chord recognition: influence of the chord types (Kobe, 2009), pp. 153-158

26. C Harte, M Sandler, in Proceedings of the 118th Convention of the Audio Engineering Society (AES). Automatic chord identification using a quantized chromagram (Spain, 2005) 
27. H Papadopoulos, G Peeters, in Proceedings of the IEEE International Conference on Acoustics, Speech and Signal Processing (ICASSP). Simultaneous estimation of chord progression and downbeats from an audio file (Las Vegas, 2008), pp. 121-124

28. L Rabiner, A tutorial on hidden Markov models and selected applications in speech recognition. Proc, IEEE. 77, 257-286 (1989)

29. DA Reynolds, RC Rose, Robust text-independent speaker identification using Gaussian mixture speaker models. IEEE Trans. Speech Audio Process. 3, 72-83 (1995)

30. T Cho, RJ Weiss, JP Bello, in Proceedings of the Sound and Music Computing Conference (SMC). Exploring common variations in state of the art chord recognition systems (Barcelona, 2010), pp. 1-8

31. SW Hainsworth, MD Macleod, Time frequency reassignment: a review and analysis Technical report, Cambridge University Engineering Department, CUED/F-INFENG/TR, 459 (June 2003)

32. F Auger, P Flandrin, Improving the readability of time-frequency and time-scale representations by the reassignment method. IEEE Trans, Speech Audio Process. 5(43), 1068-1089 (1995)

33. SA Fulop, K Fitz, Separation of components from impulses in reassigned spectrograms. J. Acoust. Soc. Am. 121(3), 1510-1518 (2007)

34. DJ Nelson, Instantaneous higher order phase derivatives. Digit Signal Process. 12(2-3), 416-428 (2002)

35. M Khadkevich, M Omologo, in Proceedings of the International Conference on Digital Audio Effects DAFx. Phase-change based tuning for automatic chord recognition (Como, 2009), pp. 506-509

36. E Gómez, Tonal description of music audio signals. PhD thesis, Universitat Pompeu Fabra (2006)

37. K Lee, in PhD thesis, Center for Computer Research in Music and Acoustics (CCRMA). A system for acoustic chord transcription and key extraction from audio using hidden Markov models trained on synthesized audio (Department of Music, Stanford University, 2008)

38. DA Reynolds, Speaker identification and verification using Gaussian mixture speaker models. Speech Commun. 17(91-108) (1995)

39. G Peeters, in Proceedings of the International Conference on Digital Audio Effects DAFx. Musical key estimation of audio signal based on HMM modeling of chroma vectors (McGill, 2006), pp. 127-131

40. C Zieger, M Omologo, in Proceedings of Interspeech. Acoustic event classification using a distributed microphone network with a GMM/SVM combined algorithm (Brisbane, 2008), pp. 115-118

41. A Klapuri, M Davy (eds.), Signal Processing Methods for Music Transcription (Springer, New York, 2006)

42. D Jurafsky, JH Martin (eds.), Speech and Language Processing: An Introduction to Natural Language Processing Computational Linguistics, and Speech Recognition (Prentice Hall Upper Saddle River, 2000)

43. C Harte, M Sandler, in Proceedings of the International Conference on Music Information Retrieval (ISMIR). Symbolic representation of musical chords: a proposed syntax for text annotations (London, 2005), pp. 66-71

44. M Mauch, S Dixon, Simultaneous estimation of chords and musical context from audio. IEEE Trans. Audio, Speech, Lang, Process. 18(6), 1280-1289 (2010)

45. C Joder, S Essid, G Richard, in Proceedings of the IEEE International Conference on Acoustics, Speech and Signal Processing (ICASSP). A comparative study of tonal acoustic features for a symbolic level music-to-score alignment (Dallas, 2010), pp. 409-412

46. M Goto, H Hashiguchi, T Nishimura, R Oka, in Proceedings of the International Conference on Music Information Retrieval (ISMIR). RWC music database: popular, classical, and jazz music databases (Paris, 2002), pp. 287-288

47. M Khadkevich, T Fillon, G Richard, M Omologo, in Proceedings of the IEEE International Conference on Acoustics, Speech and Signal Processing (ICASSP). A probabilistic approach to simultaneous extraction of beats and downbeats (Kyoto, 2012), pp. 445-448

doi:10.1186/1687-4722-2013-15

Cite this article as: Khadkevich and Omologo: Reassigned spectrum-based feature extraction for GMM-based automatic chord recognition. EURASIP Journal on Audio, Speech, and Music Processing 2013 2013:15.

\section{Submit your manuscript to a SpringerOpen ${ }^{\circ}$ journal and benefit from:}

- Convenient online submission

- Rigorous peer review

- Immediate publication on acceptance

- Open access: articles freely available online

- High visibility within the field

- Retaining the copyright to your article

Submit your next manuscript at $\boldsymbol{~ s p r i n g e r o p e n . c o m ~}$ 